Rev. Biol. Trop., 47(4): 1137-1138, 1999

www.ucr.ac.cr www.ots.ac.cr www.ots.duke.edu

\title{
Cría de murciélago en la dieta de Trachops cirrhosus (Chiroptera: Phyllostomidae) en Costa Rica
}

\author{
Vernun Arias, Federico Villalobos y José M. Mora
}

Escuela de Biología, Universidad de Costa Rica, 2060 San José, Costa Rica. Fax 207 4216. Correo electrónico jmmora@biología.ucr.ac.cr

\section{Recibido 10-XI-1988. Corregido 16-VI-1999. Aceptado 7-VII-1999.}

Key words: Artibeus, Bats, Chiroptera, Costa Rica, Feeding, Phyllostomidae, Trachops.

Trachops cirrhosus, una de las 33 especies de la subfamilia Phyllostominae (Wilson \& Reeder 1993), se distribuye desde el sur de México hasta Bolivia y el sureste de Brasil (Emmons 1990). Es un murciélago grande (71$92 \mathrm{~mm}$, con un peso de 28-42 gr.) de color pardo oscuro a gris (Mora 2000). Entre los sitios de descanso se encuentran cavernas, casas, alcantarillas, árboles huecos y túneles. Junto a otras siete especies, habita un túnel ferroviario abandonado (Miraflores), en Caldera, Puntarenas, Costa Rica. El túnel tiene una longitud de $211 \mathrm{~m}$, un ancho máximo en la base de $4.5 \mathrm{~m}$ y una altura máxima en su interior de $5.11 \mathrm{~m}$. Este lugar representa un sitio de percha además para tres especies frugívoras (Artibeus jamaicensis, Carollia perspicillata y C. castanea), una especie nectarívora (Glossophaga sorici$n a$ ), una especie hematófaga (Desmodus rotun$d u s$ ), una insectívora (Saccopterix bilineata) y una generalista (Phyllostomus discolor) (Arias \& Mora 1997; Nowak 1991). El número total de individuos de las ocho especies en el túnel se ha mantenido alrededor de 1000 individuos (Arias \& Mora 1997).

Trachops cirrhosus se alimenta especialmente de ranas aunque también incluye otros vertebrados pequeños e insectos grandes (Emmons 1990, Mora 2000). La noche del 5 de mayo de 1996 observamos a un individuo adulto de $T$. cirrhosus alimentarse de una cría de murciélago. Estaba colgado y manipulaba la presa con sus extremidades anteriores, mientras con la boca realizaba un corte a ambos lados del tronco, separando el patagio y extremidades del resto del cuerpo, con el cuál partió. Comparamos los restos caídos con la morfología del pie de otras especies presentes en el túnel. Creemos que la presa fue un $\mathrm{Arti-}$ beus jamaicensis, (este evento coincide con la época de nacimientos de esta especie frugívora en el túnel).

A partir de esta observación sería interesante cuestionarse qué importancia tiene este recurso dentro de la dieta de $T$. cirrhosus, tanto para la población presente en el túnel, como en otros sitios de descanso diurno; o si fue un comportamiento alimentario circunstancial, favorecido por la estación reproductiva de las especies frugívoras.

Este informe fue financiado por la Vicerrectoría de Investigación, Universidad de Costa Rica, bajo el proyecto No. 111-96-250 de José M. Mora. 


\section{REFERENCIAS}

Arias, V. \& J.M. Mora. 1997. Spatial distribution and population size of a bat community in a tunnel at Caldera, Costa Rica, p. 37. In Tropical Diversity: Origins, Maintenance, and Conservation. Symposium and Annual Meeting. Organización para Estudios Tropicales. San José, Costa Rica.

Emmons, L.H. 1990. NeotropicalRainforest Mammals: A field guide. University of Chicago, Chicago, Illinois. 281 p.
Mora, J.M. 2000. Los mamíferos silvestres de Costa Rica. EUNED, San José, Costa Rica (en prensa).

Nowak, R.M. 1991. Walker's Mammals of the World. Johns Hopkins University, Baltimore, Maryland. $1362 \mathrm{p}$.

Wilson, D.E. \& D.M. Reeder. 1993. Mammal species of the world. Smithsonian Institution, Washington D.C. $1206 \mathrm{p}$. 